\title{
Yod
}

Revue des études hébraïques et juives

18 | 2013

Le Proche-Orient ancien à la lumière des sciences sociales

\section{Mare nostrum : Notre mer à tous et à chacun}

The Mediterranean: Each One's Sea

Irad Malkin

Traducteur : Madalina Vârtejanu-Joubert

\section{(2) OpenEdition}

Journals

Édition électronique

URL : https://journals.openedition.org/yod/1930

DOI : $10.4000 /$ yod. 1930

ISSN : 2261-0200

Éditeur

INALCO

Édition imprimée

Date de publication : 1 septembre 2013

ISBN : 978-2-85831-207-8

ISSN : 0338-9316

Référence électronique

Irad Malkin, «Mare nostrum : Notre mer à tous et à chacun », Yod [En ligne], 18 | 2013, mis en ligne le 08 juillet 2013, consulté le 08 juillet 2021. URL : http://journals.openedition.org/yod/1930 ; DOI : https://doi.org/10.4000/yod. 1930

Ce document a été généré automatiquement le 8 juillet 2021.

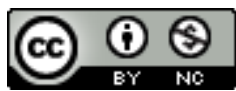

Yod est mis à disposition selon les termes de la Licence Creative Commons Attribution - Pas d'Utilisation Commerciale 4.0 International. 


\section{Mare nostrum : Notre mer à tous et à chacun}

The Mediterranean: Each One's Sea

הים שלנו ושלנו ושלנו

Irad Malkin

Traduction : Madalina Vârtejanu-Joubert

\section{NOTE DE L'ÉDITEUR}

Cet article, traduit ici de l'hébreu par Madalina Vârtejanu-Joubert, a paru dans la revue électronique « Masa Aher» Magazine 72.

1 Le hasard m'a conduit un jour, à Marseille, dans une église plongée dans la pénombre. D'une niche surgit, le visage renfrogné, un petit homme corpulent, béret bleu penché sur sa tête ovale, pipe en coin et balai de paille à la main. Il me demanda, avec un fort accent provençal, d'où je venais. "De Tel Aviv », répondis-je. Il se radoucit, plissa le front en cherchant un mot gentil qui pourrait témoigner de quelque point commun entre les Juifs d'Israël et le bedeau d'une église catholique. « Oui », finit-il par dire en souriant, "nous sommes tous les deux des Méditerranéens. " Cette rencontre survint peu après la création, par une poignée de jeunes chercheurs en histoire de l'Université de Tel Aviv - dont je faisais partie - d'une nouvelle revue internationale dédiée à l'histoire de la Méditerranée. Ainsi, les paroles de ce bedeau venaient opportunément attester du caractère durable et effectif de ce sentiment d'appartenance à l'identité méditerranéenne, tout difficile qu'il soit de définir ce sentiment avec précision. Depuis lors, et surtout durant la dernière décennie, la « méditerranéité » est devenue incontournable. En Israël, ce concept a plusieurs acceptions qui vont de son identification, condescendante et bornée, à l'«orientalité » (la «musique méditerranéenne ») à des tentatives tout à fait sérieuses de considérer la Méditerranée et le « mode de vie méditerranéen » comme une alternative significative à la notion de 
«moyen-orientalité » (c.-à-d. «arabité »), de «nord-européanité » voire même d'« américanité ».

2 L'intérêt pour la Méditerranée s'amplifie aussi à l'extérieur d'Israël, dépassant ainsi la sphère du journalisme ou de l'enseignement universitaire. Lors de la réunion de Barcelone en 1995, l'Union européenne a élaboré une charte sociale, économique et culturelle portant uniquement sur la Méditerranée et a affecté aux projets sur la Méditerranée un budget supérieur à six milliards de dollars.

De quoi s'agit-il en somme? Qu'est-ce que la Méditerranée a de spécial qui fait d'elle autre chose qu'une simple mer peuplée de poissons. Pourquoi fait-elle l'objet d'autant de productions imaginaires et de représentations, pourquoi autant de strates historiques lui sont-elles associées et pourquoi autant de processus historiques lents dont nous-mêmes ne sommes pas toujours conscients - y trouvent-ils leur aboutissement? La Méditerranée ne serait-elle qu'une invention, une abstraction d'historiens? Et quand bien même il en serait ainsi, les abstractions historiques modifient la réalité. La représentation qu'on se fait des choses, même sans rapport avec leur réalité, engendre souvent plus de conséquences que les faits solidement établis. C'est peut-être justement ce qui se passe avec la mer Méditerranée.

\section{Une lumière éclatante}

4 Élisée Reclus, géographe du XIX ${ }^{\mathrm{e}}$ siècle, étudia, parmi les premiers, la mer Méditerranée en tant que système physico-historique : « Le creuset de la civilisation et le creuset de la mer Méditerranée ne font qu'un ", affirmait-il. En Allemagne, des poètes comme Goethe et Hölderlin, mais surtout des philosophes comme Nietzsche, ont inventé un espace « gréco-méditerranéen » où coexistaient, dans un saint compromis, l'apollonien et le dionysiaque, un espace où la grâce et la danse rendent les choses plus légères et d'où surgissent la lumière et l'éclat.

5 La «lumière ", si on y prête attention, est le mot-clé qui rassemble tous ceux qui ont succombé au charme de la Méditerranée. L'emploi de ce terme trahit leur origine septentrionale, les contrées froides où le soleil est rare et où, de ce fait, les hommes percevaient l'éclat de la Méditerranée comme le signe d'une culture différente. La Méditerranée comme système est une conception qui vit le jour dans des contrées extérieures à ce système. En effet, le plus grand historien de la Méditerranée, Fernand Braudel, commence son livre en déclarant: "Je suis un homme du Nord» et la Méditerranée, située au Sud par rapport à lui, fait l'objet de sa passion.

Morris Bowra, savant réputé qui enseigne l'histoire de la Grèce classique à Oxford, explique à ses lecteurs (même non britanniques, comme il aurait convenu qu'ils le fussent), les effets d'une si forte lumière : le climat méditerranéen incite les gens à sortir de leurs maisons, à discuter, à débattre ensemble ; la lumière de la Méditerranée nous impose de voir des lignes claires et nettes et c'est ainsi que se développèrent en Grèce la démocratie (qui procède de ces rencontres dans une assemblée en plein air) et la philosophie (la production de catégories bien distinctes et d'une pensée logique).

7 La méditerranéité connaît également aujourd'hui une acception politique, procédant de l'intérieur, des habitants du pourtour méditerranéen eux-mêmes. Cependant, une fois de plus, ceux qui entendent définir à travers elle une nouvelle appartenance demeurent les minorités. Par exemple, dans les années 1920, des cercles maronites du Liban se sont 
découvert des racines phéniciennes qu'ils utilisèrent pour légitimer leur présence territoriale (à Tyr, Sidon, Byblos, etc.); ils s'appuyèrent sur les principales caractéristiques de l'antique civilisation phénicienne - l'expertise maritime, le commerce et la colonisation - ainsi que sur le savoir académique européen.

Cette vision, identifiant l'Hébreu au Phénicien, fit également son apparition au sein de cercles juifs. Comme l'a bien montré l'historien Yacov Shavit, cette vision propose de reconstituer le passé historique juif en tant que passé maritime et "d'installation ». Cette reconstitution représente en réalité l'opposé de la manière dont est attestée l'ancienne présence juive en terre d'Israël, présence qui, jusqu'à l'époque hellénistique, était coupée de la bande côtière. À mon avis, cette conception tentait surtout de fournir une justification historique à des phénomènes contemporains qui ne correspondaient pas à la manière dont les tribus d'Israël avaient pris possession de la terre dans l'Antiquité : cette fois elles n'étaient pas arrivées par le désert, ni en franchissant les montagnes, mais par des ports comme Jaffa ou Haïfa et en s'établissant le long de la côte. Le sionisme fut avant tout une expérience d'implantation côtière (méditerranéenne), tournant le regard « de l'extérieur » vers l'intérieur, vers la Terre, comme en témoigne aujourd'hui encore la concentration géographique de l'habitat juif en Israël.

Par l'invention d'un tel passé phénicien l'idée méditerranéenne se voit associée à une réalité historique préislamique. Ainsi, en Tunisie par exemple, certains courants de pensée ont développé le lien à Carthage (phénicienne elle aussi) comme référence définissant une identité méditerranéenne qui serait une alternative au panarabisme et au panislamisme. En Égypte ce furent les courants néopharaoniques qui prirent appui sur l'Égypte antique pour définir l'identité de l'Égypte moderne. On ne devrait sans doute pas s'étonner que parmi les intellectuels égyptiens porteurs de ces idées, on comptait aussi des membres de la minorité copte. En affirmant une identité égyptienne fondée non pas sur l'islam, mais sur la triade Nil-Méditerranée-Pharaons, ils espéraient parvenir à leur intégration tant souhaitée.

\section{Une cause perdue}

La plupart de ces "reconstitutions historiques" ont surtout suscité un intérêt intellectuel, débattu dans des cercles restreints, sans qu'elles prennent racine et se transforment en mythe fondateur de l'identité nationale. Mais il ne faut pas dénigrer les productions intellectuelles: c'est un fait établi que ce sont justement elles qui prodiguent à des nations entières les composantes collectivement partagées de leur identité. Et lorsque de telles conceptions prennent racine, c'est en vain qu'on peut tenter de les réfuter et de les combattre: la cause est perdue d'avance. Sous nos propres yeux l'idée méditerranéenne se propage parmi les Juifs comme parmi les Arabes et devient un mythe fondateur comme elle le fut déjà par le passé pour de nombreux mouvements d'implantation. Des sionistes aussi, dont Isaac Ben-Zvi et David Ben-Gourion, caressaient l'espoir de résoudre le conflit avec les habitants du pays en faisant appel à un dispositif intellectuel d'intégration historique. C'est ainsi, par exemple, qu'on considérait que les Arabes habitant la Palestine étaient en réalité des Juifs qui n'avaient pas connu l'exil et qui s'étaient convertis à l'islam lorsque celui-ci commença à se répandre. De cette manière, la seule chose à faire était de leur expliquer 
qui ils étaient "en réalité » et tout allait rentrer dans l'ordre. Peu se laissèrent convaincre cependant.

De l'autre côté, les Palestiniens se considéraient, déjà dans les années 30, comme les descendants des tout premiers habitants du pays: une ligne directe les reliait aux Périzzites, aux Jébusites, aux Hivvites et aux autres tribus cananéennes. Certes, leur nom avait changé - le mot "Palestinien» dérive du nom "Philistin», groupe méditerranéen qui peuplait la côte -, ainsi que leur langue et leur religion, mais du point de vue « ethnique » c'étaient bien eux les Cananéens. Et les sionistes étaient des envahisseurs étrangers tout comme Josué l'avait été autrefois. Il s'agit dans ce cas d'une expression des plus claires de l'argument «nous étions ici avant». Pendant longtemps celui-ci fut le fait d'une minorité, mais dans la dernière décennie il s'imposa soudain comme une composante importante et fondamentale de l'identité palestinienne et il est désormais enseigné à l'école. Dans moins d'une génération il deviendra vérité historique.

Dans l'Italie fasciste, l'idée méditerranéenne devint le catalyseur d'une vague impérialiste destructrice. Mussolini, s'appuyant sur l'idéal de la restauration de l'Empire romain, prit comme devise une expression latine qui, de fait, fut propre à l'Empire romain dans l'Antiquité : Mare nostrum, «notre mer ». Mussolini aspirait à un nouvel empire italo-romain, qui ferait revenir dans le giron de la civilisation latine les colonies d'Afrique du Nord tombées sous la coupe de l'islam. Pour comble d'ironie, Mussolini se tourna même contre l'Éthiopie chrétienne, mais s'empara aussi d'une partie de la Libye. Aujourd'hui encore les publications archéologiques les plus importantes portant sur la Libye (l'antique Cyrénaïque) sont en italien.

\section{L'uniformité méditerranéenne}

13 Les conceptions néo-latines, faisant de la mer Méditerranée le creuset nostalgique d'une gloire perdue, influencent aussi la vision actuelle des choses. Celle-ci ambitionne avec une certaine condescendance d'intégrer la "rive méridionale", autrement dit l'Afrique du Nord musulmane, dans le système culturel méditerranéen. Du côté arabe aussi, se manifeste le désir de dépasser l'obstacle culturel et mental qui sépare «les rives européenne et musulmane " de la mer. Dans son livre L'avenir de la culture en Égypte, Taha Hussein tente de mettre la Méditerranée au cœur de la pensée arabo-musulmane : «Les sources grecques de la culture arabe sont contrebalancées par les sources arabes de la culture européenne. C'est l'imbrication de cet héritage qui conduira au fleurissement d'une pensée méditerranéenne nouvelle." Ils sont peu nombreux à entendre ces voix. Il semble que la Déclaration européenne de Barcelone s'efforce de réparer les choses et de jeter des ponts entre les deux rives de la Méditerranée. Toujours est-il que les cyniques disent déjà que l'Europe désire mettre en avant l'identité et le fond communs méditerranéens, principalement pour empêcher les vagues migratoires du Maghreb.

Le néolatinisme fait valoir tout un pan de l'histoire méditerranéenne qui, sur un plan plus large, représente une période d'apogée dans l'histoire de l'humanité. Sans aucun rapport avec le fascisme, la période romaine fut la seule où le bassin méditerranéen se trouva sous la coupe d'une seule puissance. Pour évoquer l'uniformité méditerranéenne qui en résulta, laissons-nous conduire par un guide professionnel, auteur d'un livre 
censé accompagner les voyageurs, un Grec du nom de Pausanias, contemporain de la pax romana, au II $^{\mathrm{e}}$ siècle de notre ère.

Pour Pausanias, un voyageur qui s'embarque dans un port d'Italie pour l'Afrique du Nord, l'Espagne, la Grèce ou l'Anatolie, accostera dans des villes et des ports très semblables par leur architecture et leur mode d'organisation sociale et politique. Les élites y seront semblables et communiqueront dans une langue connue de tous, le grec ou le latin. Les bâtiments publics et les fonctions auxquelles ils sont destinés, les temples à colonnades, les statues et les peintures, les rituels auront en partage le même univers mental.

Il va de soi qu'uniformité ne signifiait pas nécessairement unité. Pausanias écrivait, tout compte fait, pour des voyageurs qui ne souhaitaient pas voir ailleurs exactement les mêmes choses qu'ils trouvaient chez eux. Pourtant, son œuvre extraordinairement riche en descriptions de monuments (datant déjà à son époque de plusieurs siècles) illustre le fait que les habitants du pourtour méditerranéen souhaitaient trouver dans le monde la singularité dans le connu, le différent dans le semblable, mais non l'étrange ou l'insolite, non «l'altérité absolue ».

\section{La force de la mythologie}

17 À l'époque gréco-romaine se généralise le procédé qui consiste à recourir à un schéma mythologique analogue pour définir l'identité de différents peuples. Déterminer « l'origine des peuples » (origines gentium) était une préoccupation savante bien établie, débouchant sur une source quasiment identique : les héros de la mythologie grecque en général et ceux de la guerre de Troie en particulier. Le cadre narratif était identique, même s'il y avait place pour la manipulation.

Selon ce schéma mythologique, tous les vaillants guerriers de la Grèce assiégèrent la ville de Troie pour ramener Hélène, la plus belle des femmes, au roi Ménélas. À la fin de cette guerre sanglante, la ville de Troie fut complètement détruite et la mer Méditerranée se remplit de rescapés troyens et de héros grecs dont le retour fut retardé par la colère des dieux. La guerre de Troie était considérée comme le Big Bang de l'Histoire : les héros de la guerre avaient fondé des villes et des dynasties sur les rives de l'Épire, de l'Italie, de l'Anatolie et du nord de l'Afrique. Avec l'essor de la colonisation grecque, ces mythes se sont répandus dans des territoires de plus en plus éloignés.

19 Les Romains ont adopté ce modèle même si, en vue d'accentuer leur spécificité par rapport aux Grecs, ils ont choisi « le côté troyen » et ont considéré le prince rescapé de Troie, Énée, comme leur fondateur (ses exploits ont été chantés, comme nous le savons, par Virgile). Les siècles ont passé et de nouveaux peuples arrivés sur le pourtour méditerranéen continuèrent à définir leur appartenance à cet espace en faisant appel aux mêmes dispositifs mythologiques. En 632, le roi des Francs, Dagobert, déclarait: «Je descends de Priam!» (roi de Troie, beau-père d'Énée). Au viII siècle, même les Anglais, comme plusieurs autres peuples, se sont insérés dans le mythe méditerranéen de Troie, à travers la descendance d'un autre prince, Brutus.

20 La chrétienté a produit elle aussi un tel modèle mental d'appartenance. Elle s'affirma clairement comme une religion méditerranéenne dans la mesure où elle a contribué de façon décisive à la préservation de l'unité « romaine » sur le plan mental et ce, même 
après la division de l'Empire romain entre l'Occident et l'Orient (Byzance), voire même après les conquêtes musulmanes. L'Église et l'Empire furent « romains » et le restèrent après le Moyen Âge, lorsque l'empereur était français ou allemand. Il en fut de même au Moyen-Orient: jusqu'à l'époque moderne les «Grecs » s'appelaient eux-mêmes, et furent appelés ensuite par les Ottomans, "Romains» (Rum, Rumli). Ce ne fut qu'avec l'essor des mouvements nationaux modernes que l'idée de Grèce (Hellas) fit son retour comme mode de définition de l'identité collective nationale (et non pas romaine ou religieuse).

Il est intéressant de noter, par exemple, comment, en dépit des changements considérables subis par la mer Méditerranée du viII ${ }^{e}$ siècle avant $n$. è. au viII ${ }^{e}$ siècle de $n$. è. - la colonisation grecque, l'essor et le déclin de l'Empire romain, l'essor de Byzance et l'invasion des peuples germaniques - les formes mentales et les modalités d'autodéfinition ont, en dépit de leurs variations, entretenu la continuité identitaire fondée sur une mythologie commune.

\section{L'éloignement de la mer}

Quand la mer Méditerranée a-t-elle, de fait, cessé de fonctionner comme «notre mer » ? Henri Pirenne, savant belge auteur d'une thèse très célèbre qui porte son nom, considère que le Moyen Âge commence seulement avec la conquête musulmane. La Méditerranée a alors été séparée en une rive sud - s'étendant du Moyen-Orient à l'Espagne, en passant par l'Afrique du Nord - et une rive nord, "européenne » en Occident. À la suite de quoi, affirme Pirenne, le centre de gravité s'est déplacé du réseau des liens constitués autour de le Méditerranée vers l'intérieur des terres, vers le Nord. L'Europe du Moyen Âge (et celle d'aujourd'hui aussi) alla de pair avec le partage de la Méditerranée entre « Mahomet et Charlemagne ».

Un grand débat a suivi cette thèse. La Méditerranée était-elle vraiment divisée ? Les recherches impressionnantes de Shlomo Dov Goitein à partir des documents de la genizah de la synagogue Ben-Ezra du Vieux Caire (Fustat), témoignent du caractère problématique de certains jugements définitifs qui tentent d'expliquer l'histoire de la Méditerranée. Dans son livre Mediterranean Society, Goitein se penche sur les documents de la genizah et dévoile une image surprenante des réseaux méditerranéens entre le $\mathrm{XI}^{\mathrm{e}}$ et le XIII ${ }^{\mathrm{e}}$ siècle, justement à l'époque où la Méditerranée était, selon Pirenne, divisée en deux.

Goitein se voyait comme un "sociographe", transcrivant la réalité au niveau microsociétal, car son travail s'appuie sur une analyse minutieuse, philologique et historique, des sources. Il montre par exemple que les Juifs n'avaient pas le monopole du commerce méditerranéen : les échanges commerciaux entre les pays chrétiens (Rum, "Rome ») et les pays islamiques se réalisaient essentiellement entre chrétiens et musulmans. La riche correspondance entre les Juifs portait essentiellement sur des sujets de société, sur la vie privée (le mariage, par exemple) et même sur la halakha. Elle reliait les deux rives de la mer sans corrélation nécessaire avec la religion dominante dans les pays où résidaient les Juifs. À cette époque, la communauté juive faisait partie intégrante de la société des pays de résidence (les ghettos n'apparurent que plus tard) et ce fut également l'époque (de Saadia Gaon à Maïmonide) où la halakha, la prière et le piyyut se développèrent. Le mode de vie des Juifs avait changé : on était passé de la prééminence de l'agriculture comme à l'époque de la Michna et du Talmud au 
commerce, à l'artisanat et aux professions libérales. Des travaux de Goitein il ressort qu'au Moyen Âge les Juifs étaient réellement un peuple méditerranéen tout comme ils l'avaient été à l'époque hellénistique et romaine.

La Méditerranée était appelée dans les manuscrits de la genizah «la Mer » ou « la mer Salée ", pour la distinguer du Nil. Cette appellation laisse supposer une certaine intimité, plus accentuée que celle qu'entretiennent les géographes musulmans qui l'appellent "la mer des Romains », Baḥr al-Rūm. La civilisation méditerranéenne se caractérisait d'une manière générale par une unité qui se fondait encore sur des racines romaines et sur le développement urbain et commercial. Contrairement à Pirenne, Goitein considérait que les débuts de la scission entre les deux rives de la Méditerranée étaient à chercher dans la conquête des pays musulmans par les peuples venus d'Asie Centrale et du Caucase. L'accession au pouvoir des Mamelouks en Égypte (1250) et les conséquences des Croisades ont modifié le rapport à la mer. Sur les rives d'Israël, on peut constater visuellement la chose : sur la côte d'Herzliya (Sidna Ali), par exemple, la plage et l'eau sont parsemées de gigantesques blocs détachés des remparts détruits et projetés depuis la crête de grès. C'est le sultan Baybars qui a détruit de manière systématique les fortifications bordant la mer, visant clairement à arrêter son plus grand ennemi: la mer elle-même par laquelle étaient constamment arrivés les envahisseurs venus d'Europe.

\section{Les mouvements de fond}

L'historien qui a le plus influencé notre vision historique de la mer Méditerranée est Fernand Braudel. Ironie de l'histoire, Goitein, contemporain de Braudel avec lequel il était en relation épistolaire, ne lut l'ouvrage de celui-ci qu'après avoir terminé sa propre recherche "et j'ai immédiatement regretté de ne pas l'avoir fait plus tôt", dit-il. Il faut préciser que Braudel est considéré comme l'un des plus grands historiens et que ses analyses portent non seulement sur la Méditerranée, mais aussi sur notre manière de concevoir l'Histoire.

Braudel témoigne de son amour de la Méditerranée et de son espoir que la lumière du soleil méditerranéen se réfracte dans les pages de son ouvrage monumental, La Méditerranée et le monde méditerranéen à l'époque de Philippe II ( $2^{\mathrm{e}}$ édition, 1966). Le premier volume ne traite pas du $\mathrm{XvI}^{\mathrm{e}}$ siècle, mais de la Méditerranée elle-même; non pas des actions des princes et des nobles, des « futilités du passé » et des « détails de la mosaïque », mais des mouvements de fond de la vie sur le pourtour méditerranéen, de "la marche lente et puissante de l'histoire ». Braudel critique les considérations géographiques qui introduisent de nombreux livres d'histoire, mais dont « on n'entend plus parler par la suite». Pour lui, la géographie, le climat, les matrices traditionnelles qui constituent l'infrastructure sur une durée plus longue que celle des changements politiques - tous ces éléments ne représentent pas le contexte pittoresque de l'histoire humaine, mais sont au contraire le cœur du sujet.

La Méditerranée de Braudel s'inscrit dans l'histoire, mais pas une histoire événementielle, avec ses dates et ses batailles, ses assassinats et ses alliances. Car que sont les "événements", s'interroge-t-il, sinon l'écume des vagues qui se soulèvent au-dessus des profondeurs de l'histoire? Nous devons apprendre à nous méfier d'une telle histoire, car elle s'avère très sensible et pleine de vibrations nerveuses qui remplissent les vies brèves des hommes et leurs courtes vues. Il faut donc apprendre à 
penser le temps historique différemment et distinguer le temps social, le temps géographique et le temps individuel ou à défaut, fragmenter l'homme en de nombreuses entités indépendantes ayant de multiples temporalités.

Qu'est-ce donc que le temps historique ? En partant de son analyse de la Méditerranée, Braudel a forgé un concept que tout historien actuel utilise: "la longue durée ». De quoi s'agit-il? Braudel propose de partir de la photographie aérienne des champs cultivés en France. Regardez, dit-il, cette division des terres qui a perduré pendant des siècles, c'est elle qui a déterminé le fonctionnement de la vie humaine, c'est elle qui a engendré les relations de dépendance et les privilèges, la richesse et la pauvreté, parfois jusqu'à nos jours. La démarcation des champs demeure valable depuis le Moyen Âge jusqu'au milieu du $\mathrm{xx}^{\mathrm{e}}$ siècle, en passant par la Renaissance et la Révolution française, et influence la vie des individus ainsi que les «structures » (autre mot clé) de leur vie sociale et économique, beaucoup plus que les événements, "l'écume des vagues », ces sujets relativement triviaux. C'est cela la «longue durée»: le temps des constructions historiques - «les profondeurs de la mer »- qui bougent lentement et selon des rythmes spécifiques. Ce sont des forces extrêmement puissantes qu'il faut évaluer et examiner selon des critères différents de ceux auxquels nous sommes habitués.

\section{Continuité et changement}

30 La longue durée est à la fois objet d'étude et instrument de compréhension historique. Dans la perspective d'une « historie globale» développée par Braudel, on a besoin de « ralentissement » pour se représenter les structures du temps long. C'est au fond le regret de tous les historiens : ils sont limités dans leur capacité humaine à saisir les choses comme des images à un moment donné (synchronisme). Et puisque l'histoire a également pour objet les changements et les processus (diachronie), ces phénomènes font que l'image est morcelée en un ensemble d'éléments qui changent chacun selon un rythme propre. Le philosophe grec Héraclite affirma qu'on ne peut pas se baigner deux fois dans la même rivière, car celle-ci s'écoule sans cesse et elle n'est donc à aucun moment la «même » rivière. D'un autre côté, nous savons tous, ne serait-ce que de façon intuitive, qu'il y a une continuité dans l'histoire. La tension entre continuité et changement représente l'un des problèmes les plus anciens et les plus fascinants de l'histoire.

31 Braudel le résout dans la perspective de l'histoire méditerranéenne en distinguant plusieurs dimensions du temps historique : long, intermédiaire et court. Son livre suit cette même division: la première partie traite du temps long, de la géographie (" depuis, au nord, la limite où poussent les oliviers jusqu'à la limite méridionale des bosquets de palmiers »), du climat, des routes terrestres et maritimes, des villes, etc. La deuxième partie aborde le temps intermédiaire et présente les « destins collectifs et les mouvements d'ensemble ", autrement dit l'économie et la démographie.

Dans la troisième partie, Braudel aborde le temps court. C'est là qu'il présente de fait ses travaux sur la Méditerranée effectués pendant sa jeunesse: la Méditerranée à l'époque de Philippe II. En lisant cette partie, le lecteur comprend de manière beaucoup plus approfondie l'histoire événementielle, car l'histoire méditerranéenne est intégrée dans les différentes échelles de temps. Plus que toute autre mer, la Méditerranée se prête le mieux à ce type de recherche historique. Le motto de Braudel - «Jusqu'à 
présent on n'a découvert dans les îles indiennes aucune mer Méditerranéenne comme il existe en Europe, en Asie et en Afrique »- est une citation d'Uriel da Costa datant de 1604.

L'interrelation de l'espace (ce que Braudel appelle «structure ») et du temps (en réalité «les temps »), vue jusque-là comme une contradiction extrême, reçoit une nouvelle interprétation à la lumière de l'approche braudélienne. Par exemple, des phénomènes semblables, par delà des frontières religieuses ou linguistiques, trouvent finalement une explication.

Nous avons vu plus haut la pérennité multiforme du concept de "Rome » et la continuité des mythes identitaires. La question méditerranéenne peut être posée de nombreuses autres manières. Par exemple, pourquoi les femmes s'habillent-elles en noir dans la Grèce orthodoxe et dans la Sicile et l'Espagne catholique ? Pourquoi le phénomène du patronage est-il si développé en Europe du Sud, comme l'a bien mis en évidence l'historien Gabriel Herman, et jusqu'où doit-on remonter dans le temps pour en trouver les racines? Est-ce que les sociétés méditerranéennes fonctionnent selon le modèle de l'opposition "honneur - honte", comme le pense l'anthropologue Peristiany ${ }^{1}$ ? Pourquoi Chypre, comme l'a montré Benjamin Arbel, a-t-elle conservé ses formes de pouvoir même après le passage de la domination vénitienne à la domination ottomane?

Dans son livre La liberté ou la mort, l'écrivain grec Nikos Kazantzakis confronte le Capitaine Mihalis, le patriote, au gouverneur turc Nouri Bey. La différence entre les deux est abyssale: l'un est chrétien, l'autre musulman, l'un est implacable et impétueux, l'autre gros et jouisseur, l'un lutte pour la libération de la Crète, l'autre incarne son asservissement. Pourtant Kazantzakis, lui-même d'origine crétoise, dessine des personnages étonnamment semblables en ce qui concerne leur représentation de la virilité, de l'honneur, de la domination sur les femmes, etc. Ces conceptions traversent les espaces, ne tiennent compte ni de la religion, ni de l'origine ethnique et leurs racines se perdent dans la nuit des temps.

La Méditerranée n'est pas seulement la mer où «fleurissent les citronniers » (Goethe), où poussent le basilic et les tomates. Ce n'est pas uniquement la mer sur les rives de laquelle on passe sa vie dans les cafés, au soleil, où le contact est plus intime, plus chaleureux et plus bruyant. C'est une mer avec une histoire commune qui relie ses deux rives, que nous en soyons conscients ou pas. Il serait bon que le lecteur israélien, dont le mode de vie est bien plus proche de celui d'un Grec, d'un Italien ou d'un Espagnol que de celui d'un Suisse, d'un Hollandais ou d'un Danois, s'en rende compte.

\section{NOTES}

1. J. G. Peristiany (éd.), Honor and Shame: Values of Mediterranean Society, Londres, Weidenfeld \& Nicolson, 1965. 


\section{RÉSUMÉS}

La Méditerranée a-t-elle quelque chose de particulier? Pourquoi fait-velle l'objet d'autant de productions imaginaires et de représentations? N'est-elle qu'une invention, une abstraction d'historiens? Notre article présente l'histoire des représentations de la Méditerranée ainsi que de la prise de conscience de son existence en tant qu'espace unifié.

What is so special about the Mediterranean Sea? Why is it subject of so many representations and imaginary creations? Could it be that the Mediterranean is only an invention, an historian's abstraction? This article presents the history of the Mediterranean representations as a geo-political unit.

INDEX

מילות מפתח

מיתולוגיה ים תיכונית, הצגתו של הים התיכון, המזרח התיכון, הים התיכון:

Keywords : Mediterranean mythology, representation of the Mediterranean, Mediterranean, Middle East

Mots-clés : mythologie méditerranéenne, représentation de la Méditerranée

Index géographique : Méditerranée, Proche-Orient

\section{AUTEURS}

IRAD MALKIN

Université de Tel Aviv 